\title{
Geometric Singularities and Spectra of Landau-Ginzburg Models
}

\author{
B. R. Greene' ${ }^{1}$, S.-S. Roan ${ }^{2}$ and S.-T. Yau ${ }^{3}$ \\ 1 Newman Laboratory of Nuclear Studies Cornell University, Ithaca, NY 14853, USA \\ ${ }^{2}$ Max-Planck Institut für Mathematik Gottfried-Claren-Straße 26 W-5300 Bonn 3, Federal \\ Republic of Germany \\ 3 Department of Mathematics, Harvard University, Cambridge, MA 02138, USA
}

Received July 9, 1990; in revised form January 4, 1991

\begin{abstract}
Some mathematical and physical aspects of superconformal string compactification in weighted projective space are discussed. In particular, we recast the path integral argument establishing the connection between LandauGinzburg conformal theories and Calabi-Yau string compactification in a geometric framework. We then prove that the naive expression for the vanishing of the first Chern class for a complete intersection (adopted from the smooth case) is sufficient to ensure that the resulting variety, which is generically singular, can be resolved to a smooth Calabi-Yau space. This justifies much analysis which has recently been expended on the study of Landau-Ginzburg models. Furthermore, we derive some simple formulae for the determination of the Witten index in these theories which are complimentary to those derived using semiclassical reasoning by Vafa. Finally, we also comment on the possible geometrical significance of unorbifolded Landau-Ginzburg theories.
\end{abstract}

\section{Introduction}

During the past year much progress has been made in understanding the previous mysterious connection between minimal model string vacua [1] and geometrical compactification on manifolds with trivial canonical bundle $[2,3]$. The unifying link between the two is a Landau-Ginzburg description of the minimal model theories which gives rise, with little difficulty, to a nonlinear sigma model with Calabi-Yau target space. In particular, an orbifold of the Landau-Ginzburg theory lies in the same universality class as a string propagating in a Calabi-Yau background embedded in weighted projective space. Weighted projective space, unlike ordinary projective space, has quotient singularities. Hence, a generic Calabi-Yau manifold embedded in such a background has such singularities as well. 
An important and well known property of string propagation in singular backgrounds [4] is that the string probes the topology of the desingularized counterpart of the original space which shares the property of trivial canonical bundle. Physically, this property manifests itself, for example, in the fact that it is the cohomology of the desingularized manifold (as opposed to that of the singular space) which determines the spectrum of massless excitations. In the context of minimal model vacua, this feature has been used to verify in explicit examples that the general arguments of [2] correctly pair these minimal model theories with geometrical backgrounds. That is, the minimal model spectra have been compared and shown to agree $[2,5,6,7]$ with the cohomology of the desingularized Calabi-Yau manifolds dictated by $[2,3]$.

All of these analyses, though, make one crucial assumption: the singularities encountered in these Calabi-Yau constructions are such that a desingularization exists. Under this nontrivial assumption, the authors above have made use of the procedure described in [2] as adapted from [8] to compute the properties of the resolved space. Physically one expects this assumption to be true, as emphasized in [9] and [3], since the Landau-Ginzburg theories give rise to well defined string vacua and the latter, if singular, admit smooth resolutions [4]. It is one purpose of this letter, to rigorously prove that in the cases under consideration, a smooth resolution to a smooth manifold of trivial canonical bundle always exists.

In addition, there are three other issues we shall discuss. First, we shall briefly rephrase the analysis of [2] in a way that makes the geometrical underpinnings of the arguments more apparent. Second, we shall derive some general formula for the Euler number of the resolved weighted projective space Calabi-Yau constructions under consideration. This provides a more general and systematic method of computation compared with the procedure described and applied in $[2]^{1}$. Finally, we shall briefly describe a possible geometric interpretation for Landau-Ginzburg theories in contradistinction to Landau-Ginzburg orbifolds which correspond to strings propagation on Calabi-Yau manifolds. This is a question which deserves further study.

\section{Landau-Ginzburg Effective Theories and Geometric String Propagation}

In this section we briefly review the results of [2] and [3] regarding the connection between Landau-Ginzburg effective Lagrangians and string propagation on Calabi-Yau manifolds. We rephrase the treatment in [2] in a way that exposes the underlying geometrical nature of the constructions utilized.

The connection between $N=2$ minimal model vacua and geometric string propagation, as conjectured in [1] and established in $[2,3]$, is based upon the observation [11] that the $p^{\text {th }}$ minimal model has a Lagrangian description with superpotential $\Phi^{P+2}$. The field $\Phi$ is a two dimensional chiral superfield. A tensor

\footnotetext{
${ }^{1}$ Recently, Vafa [10] has employed a semiclassical argument in the Landau-Ginzburg formalism to derive similar formulas for these Euler numbers. As will be shown elsewhere, the results of the present paper can be extended to prove Vafa's result and hence establish a precise connection between blow-up modes and resolved cohomology classes
} 
product of $r$ minimal models, $\left(P_{1}, \ldots, P_{r}\right)$ is thus described by a superpotential

$$
W=\Phi_{1}^{P_{1}+2}+\cdots+\Phi_{r}^{P_{r}+2} .
$$

The Lagrangian for this theory can thus be written

$$
\int d^{2} z d^{4} \theta K\left(\bar{\Phi}_{i}, \Phi_{i}\right)+\left(\int d^{2} z d^{2} \theta W\left(\Phi_{i}\right)+\text { c.c }\right) \text {. }
$$

As described in [12] the renormalization group flow for such a theory is driven by the superpotential, $W$, as the $D$ terms supply irrelevant perturbations. In fact, $W$ is not renormalized and hence provides us with an invariant of the renormalization group flow with which to characterize such 2 -dimensional theories [12]. Following [2], we move to a point on the renormalization group trajectory with infinitesimal $D$-terms and hence describe the theory with the path integral

$$
\int\left[d \Phi_{1}\right] \cdots\left[d \Phi_{5}\right] \exp \left\{i \int d^{2} z d^{2} \theta\left(\Phi_{1}^{l_{1}}+\cdots \Phi_{5}^{l_{5}}\right)\right\},
$$

where for definiteness we have restricted our attention to five complex variables and set $l_{i}=P_{i}+2$ for ease of notation. Now, notice that if we consider an orbifold of this Landau-Ginzburg theory

$$
\left(\Phi_{1}, \ldots, \Phi_{5}\right) \sim\left(e^{2 \pi i / l_{1}} \Phi_{1}, \ldots, e^{2 \pi i / l_{5}} \Phi_{5}\right),
$$

we are justified in performing the nonlinear change of variables from $\left(\Phi_{1}, \ldots, \Phi_{5}\right)$ to $\left(\xi_{1}, \ldots, \xi_{5}\right)$ where

$$
\xi_{1}=\Phi_{1}^{l_{1}} \quad \xi_{i}^{l_{i}}=\frac{\Phi_{i}^{l_{i}}}{\Phi_{1}^{l_{1}}}
$$

In terms of these new variables, we can write the path integral (2.3) as

$$
\int\left[d \xi_{1}\right] \cdots\left[d \xi_{5}\right] \Omega \exp \left\{i \int d^{2} z d^{2} \theta \xi_{1}\left(1+\xi_{2}^{l_{2}}+\cdots \xi_{5}^{l_{5}}\right)\right\},
$$

where $\Omega$ is the Jacobian for this coordinate transformation. In particular, this Jacobian is easily seen to be proportional to $\Phi_{1}^{j}$ where $j=1-l_{1}+l_{1}\left(\sum_{i=2}^{5} \frac{1}{l_{i}}\right)$. The
crucial point is that $j$ vanishes precisely if

$$
\sum_{i=1}^{5} \frac{1}{l_{i}}=1
$$

When this condition is met, we can perform the $\xi_{1}$ integration in (2.6) to yield a multiplicand

$$
\delta\left(1+\xi_{2}^{l_{2}}+\cdots \xi_{5}^{l_{5}}\right) .
$$

Interpreting the scalar component of the superfields as background spatial coordinates, we thus see that the above delta function constrains classical motion of the string to lie on the hypersurface defined by the vanishing locus of the argument of the delta function ${ }^{2}$. As shown in [2], (2.7) is precisely the requirement that this hypersurface has vanishing first Chern class and hence is

\footnotetext{
${ }^{2}$ The $\theta$ component of the delta function constrains the fermionic superfield components to be tangent to this manifold
} 
the Calabi-Yau manifold $\sum_{i=1}^{5} z_{i}^{l_{i}}=0$ in $W C P_{\left(d / l_{1}\right), \ldots,\left(d / l_{5}\right)}^{4}$. Thus, we see that an orbifold of the original Landau-Ginzburg theory lies in the same universality class as string propagation on a Calabi-Yau manifold.

An important element of the above reasoning involves ensuring that the change of variables does not introduce a nontrivial Jacobian. This suggests that the argument can be rephrased as the existence of some global geometric structure since the triviality of a Jacobian reflects the fact that there is a trivial transformation law for an object from one coordinate patch to another. We now rephrase the above argument making use of such ideas.

To be concrete, we will phrase our argument through the simplest example the Calabi-Yau manifold $Y_{4 ; 5}$ defined by $z_{1}^{5}+z_{2}^{5}+z_{3}^{5}+z_{4}^{5}+z_{5}^{5}=0$, in $C P^{4}$, although our remarks are quite general.

Suppressing superspace dependence, the chiral fields $z_{1}, \ldots, z_{5}$ span $C^{5}-[0]$. We can think of the latter as being the line bundle $H^{-1}-[0]$ over $C P^{4}$, where $H$ is the usual hyperplane bundle. In our argument above, the orbifold operation we perform is $\left(z_{1}, \ldots, z_{5}\right) \rightarrow\left(\lambda z_{1}, \ldots, \lambda z_{5}\right)$, where $\lambda$ is a nontrivial fifth root of unity. Thus, we are mapping the configuration space $C^{5}-[0]$ to $\left(C^{5}-[0]\right) / Z_{5}$ by this action. The latter is equivalent to the line bundle $H^{-5}-[0]$ over $C P^{4}$. To be concrete, let us spell this out in a bit more detail. We choose to work over the patch $z_{1} \neq 0$ in $C P^{4}$ which has local coordinates $\left(\xi_{2}, \ldots, \xi_{5}\right)$. Local coordinates on the bundle $H^{-1}-[0]$ over $C P^{4}$ can be taken as $\left(z_{1} ; \xi_{2}, \ldots, \xi_{5}\right)$ with $z_{1}$ being the fibre coordinate. Now, the $Z_{5}$ action on these coordinates is simple $\left(z_{1} ; \xi_{2}, \ldots, \xi_{5}\right)$. $\rightarrow\left(\lambda z_{1} ; \xi_{2}, \ldots, \xi_{5}\right)$. Thus, $\left(C^{5}-[0]\right) / Z_{5}$ has local coordinates $\left(\mu ; \xi_{2}, \ldots, \xi_{5}\right)$ with $\mu=z_{1}^{5}$. Following the notation of [2], we relabel $\mu=\xi_{1}$. Clearly, then, $\left(C^{5}-[0]\right) / Z_{5}$ is equivalent to the line bundle $H^{-5}-[0]$. Note also that nothing prevents us from extending the fiber over our line bundle to get $H^{-5}$ over $C P^{4}$. We thus see that the latter is the true configuration space for the orbifolded Landau-Ginzburg theory.

Having identified the correct configuration space, let us return to the issues involved in the path integral. After changing variables as described above [2], the integration measure we use is $d \xi_{1} \wedge \cdots \wedge d \xi_{5}$ and the important point is that the Jacobian from the $z_{i}$ to the $\xi_{i}$ is a constant. To understand the significance of this, let us work, again, in the patch $z_{1} \neq 0$ and consider the measure on the total space of line bundle $H^{-5}-[0]$ over $C P^{4}$. This is simple $d \xi_{1} \wedge \cdots \wedge d \xi_{5}$. Now, what form does this measure take if we move on to the patch, say, where $z_{2} \neq 0$ ? It is simple to see that in this patch the measure still takes the form $d \xi_{1} \wedge \cdots \wedge d \xi_{5}$. In other words, the fact noticed and utilized in [2], regarding constant Jacobian change of variables, is the geometric statement that the total space of the bundle $H^{-5}-[0]$ over $C P^{4}$ admits a global measure. We call this total space $T H(-5)$. Now, with this global measure we can consistently consider

$$
\int_{T H(-5)} d \xi_{1} \wedge \cdots \wedge d \xi_{5} e^{i \tilde{f}}
$$

so long as $\tilde{f} \in \Gamma\left(C P^{4}, H^{5}\right)$. Explicitly, then, $\tilde{f}=a \xi_{1}+\xi_{1}\left(P_{5}\left(\xi_{2}, \ldots, \xi_{5}\right)\right)$, where $P_{5}$ is any fifth order polynomial. For example, we can take $f=\xi_{1}\left(1+\xi_{2}^{5}+\cdots+\xi_{5}^{5}\right)$, thus reproducing the argument in [2]. Notice that were we to try this procedure for $T H(-n)$ with $n \neq 5$, we would not be able to construct a global measure, and 
the argument would fail. Thus, the condition for vanishing first Chern class is equivalent to the existence of a global measure. This in turn allows for a simple evaluation of the path integral.

Clearly, this argument is quite general. Complete intersections can be expressed as the zero section of (tensor products) of hyperplane bundles over (weighted) projective spaces. Such sections can be integrated over the total space of the dual bundle provided we have a global measure on the latter. The existence of such a measure is precisely equivalent to the complete intersection having vanishing first Chern class - i.e. being Calabi-Yau.

This, then, is the geometrical content of the method proposed and utilized in [2].

\section{Varieties in Weighted Projective Space}

In this section we shall investigate the conditions under which a singular CalabiYau variety in weighted projective space can be resolved to a smooth CalabiYau manifold. To orient the discussion, two remarks are in order. First, we recall that a similar question has been studied in [8]. In these works it was shown that

Theorem. If $X$ is a (possibly singular) algebraic variety of dimension two or three with (at most) abelian quotient singularities, and the canonical sheaf $\omega_{X}$ of $X$ is trivial, then there exists a resolution $\tilde{X}$ of $X$ with $\tilde{X}$ a smooth variety with trivial canonical bundle.

In other words, if $X$ has trivial canonical bundle away from its singularities, then these singularities can be repaired without spoiling the triviality of the canonical bundle. Our task, therefore, will be to determine when a complete intersection in a product of weighted projective spaces satisfies this requirement.

Second, to be concrete, we remind the reader of the origin of singularities in these spaces. In $W C P_{k_{1}, \ldots, k_{N+1}}^{N}$, we make the identification $\left[z_{1}, \ldots, z_{N+1}\right] \equiv$ $\left[\lambda^{k_{1}} z_{1}, \ldots, \lambda^{k_{N+1}} z_{N+1}\right]$. If any of the $k_{i}$ have a nontrivial common factor, say for $i \in I$, then there are clearly fixed points under the above equivalence relation of form $z_{j}=0$ for $j \in I$, and $\lambda^{q}=1$ for $q$ dividing the $k_{i}, i \in I$. It is these cyclic quotient singularities which shall be our interest.

As in the case of complete intersections in products of ordinary projective spaces, it is useful to introduce a handy piece of notation called the configuration matrix [13]. This is a matrix of dimension $l$ by $k$, where $l$ equals the number of weighted projective spaces whose Cartesian product forms the ambient space and $k$ equals the codimension of the variety being described. The $(i, j)$ entry of this matrix, $d_{i j}$ is the degree of the $j^{\text {th }}$ equation in the $i^{\text {th }}$ weighted projective space. For the case of nonsingular varieties in products of ordinary projective spaces, the condition on the configuration matrix for it to describe a manifold of vanishing first Chern class is for all $i$ : [13].

$$
N_{i}+1=\sum_{j=1}^{m} d_{i j}
$$

This is easily proved from the adjunction formula. The corresponding formula for smooth complete intersections in products of weighted projective spaces, 
again easily derived from the adjunction formula, is for all $i$ :

$$
\sum_{j=1}^{N_{i}+1} q_{j}^{(i)}=\sum_{j=1}^{m} d_{i j}
$$

where the $i^{\text {th }}$ weighted projective space is $W C P_{q_{1}^{(i)} \ldots, q_{N_{1}+1}^{(i)}}^{N_{2}}$. (When there is no loss of clarity, we suppress the (i) index.) For complete intersections of complex dimension two or three in products of weighted projective spaces which intersect the ambient space singularities and hence are not smooth, we now prove:

Theorem. If $X$ is a complete intersection in a product of weighted projective spaces which meets condition (3.2), then there exists a desingularization $\tilde{X}$ of $X$ which is smooth and has vanishing first Chern class.

As discussed above, the results of [8] reduce the proof of this statement to a computation of the canonical bundle of $X-\Sigma$, where $\Sigma$ is defined to be the set of singularities of $X$, a computation to which we now turn.

Let $M$ be an $(n+l)$ dimensional complex manifold, and let $G$ be an $l$ dimensional algebraic torus $\left(C^{*}\right)^{l}$, acting on $M$. For each $y \in M$, we assume that the isotropy subgroup $G_{y}$ (that is, the set of all $g \in G$ such that $g(y)=y$ ) is finite and there is a $G_{y}$ invariant $n$-dimensional subspace $W_{y}$ of $M$ such that $y$ is a smooth point of $W_{y}$, and $W_{y}$ meets the $G$-orbit $G(y)$ of $y$ transversely at the point $y$. (As we shall see later on, $l$ will correspond to the number of weighted projective spaces composing the ambient space, and $M$ will correspond to the affine variety associated with the projective variety under consideration.) Let $X=M / G$ and let $p: M \rightarrow X$ denote the natural projection. $X$ is clearly a $V$ manifold (that is, locally of the form (smooth manifold)/(finite group action)), with (at most) abelian quotient singularities. In fact, for $x \in X$ and $x=p(y),(X, x)$ is isomorphic to $\left(W_{y} / G_{y}, y\right)$ as germs of analytic spaces. As an example, let $l=1$, $n=3$ and $M=\left\{z \in C^{5}-\{0\} \mid z_{1}^{4}+z_{2}^{4}+z_{3}^{4}+z_{4}^{8}+z_{5}^{8}=0\right\}$. Consider the $G$-action $G \times M \rightarrow M$ given by $\left(z_{1}, \ldots, z_{5}\right) \rightarrow\left(\lambda^{2} z_{1}, \lambda^{2} z_{2}, \lambda^{2} z_{3}, \lambda z_{4}, \lambda z_{5}\right)$. Then, for $y$ of the form $\left(y_{1}, y_{2}, y_{3}, 0,0\right)$, we see that $G_{y}=Z_{2}$. For all other $y, G_{y}=I$. For the former case, we can take $W_{y}=\left\{\left(y_{1}, z_{2}, z_{3}, z_{4}, z_{5}\right) \cap M\right\}$ ( $y_{1}$ fixed), while for the latter we can taken $W_{y}=\left\{\left(z_{1}, z_{2}, z_{3}, y_{4}, z_{5}\right) \cap M\right\}\left(y_{4}\right.$ fixed). The idea is that if $G_{y}$ acts freedly, we can simply collapse the $G$-orbit of $W_{y}$ to arrive (locally) at the variety $X$. If, however, $G_{y}$ does not act freely, there are further global identifications on $W_{y}$ which must be made.

There is an important point which we should presently introduce. If $\left(G_{y}\right)_{0}$ is a subgroup of $G_{y}$ which is generated by all elements in $G_{y}$ which fix some $(n-1)$ dimensional submanifold of $W_{y}$ near $y$, then $W_{y} /\left(G_{y}\right)_{0}$ is smooth. That is, $\left(W_{y} /\left(G_{y}\right)_{0}, y\right) \equiv\left(C^{n}\right.$, origin). In other words, as far as singularities go, we can disregard the subgroup $\left(G_{y}\right)_{0}$. The reason for $W_{y} /\left(G_{y}\right)_{0}$ being nonsingular is quite simple, and is most easily understood by considering a simple involution $g: z \rightarrow-z$ in $C^{1}$ as compared with $C^{2}$. In the former case we can introduce the coordinate $w=z^{2}$ on $C^{1} / g$, and thereby see that the latter is nonsingular, even though the origin is fixed by $g$. In the case of $C^{2} / g$ we can try the coordinates $\left(w_{1}, w_{2}\right)=\left(z_{1}^{2}, z_{2}^{2}\right)$, however it is clear that these are not a good choice as both $\left(-z_{1}, z_{2}\right)$ and $\left(z_{1},-z_{2}\right)$ are identified with $\left(z_{1}, z_{2}\right)$. We are thus led to distinguish the latter points with a third coordinate $w_{3}=z_{1} z_{2}$. We now see that $C^{2} / g$ is isomorphic to $w_{3}^{2}=w_{1} w_{2}$ in $C^{3}$, which is clearly a singular space. This simple 
example captures the essential reason why group actions leaving a codimension one fixed point set do not lead to singularities. Hence, we see that for each $x \in X$, $(X, x) \equiv\left(C^{n} / \gamma,[0]\right)$, where $\gamma$ is a "small" finite abelian subgroup of $G L(n, C)$, where "small" means that no element has 1 as an eigenvalue of multiplicity exactly $n-1 . X$ is therefore normal which implies $\Gamma\left(X, O_{X}\right)=\Gamma\left(X-\Sigma, O_{X-\Sigma}\right)$, and $\Sigma$ has codimension at least two.

We define the dualizing sheaf of $X$ (which in the smooth case simply refers to the canonical bundle of $X$ ) as the pushforward of the dualizing sheaf on $X-\Sigma$, that is

$$
\omega_{X}=i_{*} \omega_{X-\Sigma}
$$

with $i: X-\Sigma \rightarrow X$ and $\omega_{x-\Sigma}=\Omega_{X-\Sigma}^{n}$.

Lemma 1. $\omega_{X}=\left(p_{*} \omega_{M}\right)^{G}$, where the latter denotes the natural projection of the $G$ invariant part of the canonical bundle on $M$.

We remark that this lemma will give us a simple means of determining when the canonical bundle of $X$ is trivial, the goal we have set out upon.

Proof. Let $M^{\prime}=M-p^{-1}(\Sigma)$. Because $X$ is a $V$-manifold we only show that

$$
\omega_{X-\Sigma}=\left(p_{*} \omega_{M^{\prime}}\right)^{G}
$$

Given $x \in X-\Sigma$ and $y \in M$ with $p(y)=x$, we have

$$
(X, x) \equiv\left(W_{y} / G_{y}, y\right) \equiv\left(C^{n} /\left(\mu_{r_{1}}, \ldots, \mu_{r_{n}}\right),[0]\right) \equiv\left(C^{n},[0]\right),
$$

where $\mu_{j}=\left\{z \in C^{*} \mid z^{j}=1\right\}$ and $\left(\mu_{r_{1}}, \ldots, \mu_{r_{n}}\right)$ acts diagonally on $C^{n}$. The final equivalence is due to our working on the nonsingular subspaces. For a small neighborhood $U$ of $x$ in $X-\Sigma$, we have

$$
U \equiv p^{-1}(U) / G \equiv\left(p^{-1}(U) / G_{y}\right) /\left(G / G_{y}\right),
$$

where $p^{-1}(U) / G_{y}$ is non-singular and is acted upon freely by $G / G_{y} \equiv\left(C^{*}\right)^{l}$. From (3.6), we have a principle bundle over $U$

$$
G / G_{y} \rightarrow p^{-1}(U) / G_{y} \rightarrow U
$$

with fibre $p^{-1}(U) / G_{y}$ (over $y \in U$ ) and structure group $G / G_{y}$, which acts freely and transitively on the fiber by the adjoint action. Associated to such a principle bundle is the well known Atiyah exact sequence of vector bundles over $U$

$$
0 \rightarrow L_{U} \rightarrow Q_{U} \rightarrow T_{U} \rightarrow 0,
$$

where $T_{U}$ is the tangent bundle over $U, Q_{U}$ is the vector bundle whose fibre at the point $x \in U$ consists of the $G / G_{y}$ invariant vectors tangent to $p^{-1}(U) / G_{y}$, that is $Q_{U, x}=\Gamma\left(p^{-1}(x) / G_{y}, T_{p^{-1}(U) / G_{y}}\right)^{G / G_{y}}$, and $L_{U}$ is the vector bundle whose fibre at the point $x \in U$ is the subset of $Q_{U, x}$ consisting of vectors which are tangent to $p^{-1}(x) / G_{y}$, that is $L_{U, x}=\Gamma\left(p^{-1}(x) / G_{y}, T_{p^{-1}(x) / G_{y}}\right)^{G / G_{y}}$. It is immediate that

and

$$
Q_{U, x} \equiv \Gamma\left(p^{-1}(x), T_{M^{\prime}}\right)^{G}
$$

$$
L_{U, x} \equiv \Gamma\left(p^{-1}(x), T_{p^{-1}(x)}\right)^{G} .
$$

By patching the sequence (3.8) over a covering $\left\{U_{i}\right\}$ of $X-\Sigma$, we arrive at the 
Atiyah exact sequence associated to the fibration $p: M^{\prime} \rightarrow X-\Sigma$

$$
0 \rightarrow L \rightarrow Q \rightarrow T_{X-\Sigma} \rightarrow 0 .
$$

Now, since $G$ is abelian, $L \equiv(X-\Sigma) \times C^{l}$. Hence $\Lambda^{n+1} Q \equiv \Lambda^{n} T_{X-\Sigma}$ and we have

$$
\left(p^{*} \omega_{M^{\prime}}\right)^{G} \equiv O\left(\Lambda^{n+1} Q^{*}\right) \equiv O\left(\Lambda^{n} T_{X-\Sigma}^{*}\right) \equiv \omega_{X-\Sigma},
$$

as was to be shown.

With this lemma in hand, we can now compute the canonical bundle for the cases of interest, so long as they meet the assumptions described earlier. We now show that algebraic subvarieties of a product of weighted projective spaces do in fact meet these requirements.

To do so, consider a linear action of the algebraic torus $G$ on an $N$-dimensional $C$-vector space $V$

$$
\rho: G \rightarrow G L(V) .
$$

We denote by $\chi_{j} \in \operatorname{Hom}\left(G, C^{*}\right)$ for $j=1, \ldots, N$ the eigenvalues of $G$ (counting multiplicity), which we call the weights of $\rho$. Regarding $V$ and $C^{N}$, we may describe the action of $g \in G$ by

$$
\left(z_{1}, z_{2}, \ldots, z_{N}\right) \rightarrow\left(\chi_{1}(g) z_{1}, \chi_{2}(g) z_{2}, \ldots, \chi_{N}(g) z_{N}\right) .
$$

Let $S$ be a $G$-invariant algebraic subvariety of $C^{N}$ such that $S-[0]$ is nonsingular and $G_{y}$ is finite for each $y \in S-[0]$. Set $M=S-[0]$. In this expression [0] denotes a union of linear subspaces of $C^{N}$ whose stabilizer is infinite. To be concrete, take for example, $N=4$ and $l=2$ with $\left(z_{1}, z_{2}, z_{3}, z_{4}\right) \rightarrow$ $\left(\lambda z_{1}, \lambda^{2} z_{2}, \mu z_{3}, \mu^{3} z_{4}\right)$. Then, $[0]=(0,0) \times C^{2} \cup C^{2} \times(0,0)$ and $M / G=W C P_{1,2}^{2} \times$ $W C P_{1,3}^{2}$. We now show that $M$ satisfies the assumptions given earlier in order to prove the lemma above.

Let $y=\left(y_{1}, \ldots, y_{N}\right)$ be an element of $M$, and assume that $y_{j} \neq 0$ for $j \leqq m$ with $m \in Z$ and $m \geqq l$. (Since $G \equiv\left(C^{*}\right)^{l}$, we are going to be considering an ambiant space composed of the product of $l$-weighted projective space factors. The $y$ are coordinates on the product of affine patches of these weighted projective spaces, and hence at least $l$ of them must be nonzero.) We directly see that $G_{y}=\cap_{j=1}^{m} \operatorname{ker} \chi_{j}$. In particular, we can find $l$ elements among $\chi_{1}, \ldots, \chi_{m}$, say $\chi_{1}, \ldots, \chi_{l}$, with $\left|\cap_{j=1}^{l} \operatorname{ker} \chi_{j}\right|<\infty$. Denote by $V_{y}$ the affine subspace of $C^{N}$ defined by $z_{1}=y_{1}, \ldots, z_{l}=y_{l}$. Then, $V_{y}$ is $G_{y}$-invariant and is transversal to the $G$-orbit of $y$ at $y$. Hence, $V_{y}$ intersects $M$ transversely at $y$. If we define $W_{y}=V_{y} \cap M$, we see that $W_{y}$ satisfies the assumptions underlying Lemma 1.

We now turn to a determination of the structure of the canonical bundle of a complete intersection in a product of weighted projective spaces. To do so, we assume from now on that the ideal $\mathscr{I}_{S}=I_{S} O_{S}$ of $S$ in $C\left[z_{1}, \ldots, z_{N}\right]$ is generated by a regular sequence of $k G$-eigenfunctions $f_{1}, \ldots, f_{k}$ in $C\left[z_{1}, \ldots, z_{N}\right]$ with $k$ being the codimension of $S$ in $C^{N}$. If $\delta_{j}$ is the eigenvalue of $f_{j}$, then we say that $S$ is a complete intersection of degree $\delta_{1}, \ldots, \delta_{k}$. By the adjunction formula,

$$
\omega_{M}=\left.\left[\omega_{C^{N}} \otimes\left(\Lambda^{k} \mathscr{I}_{S}\right)^{-1}\right] \otimes O_{S}\right|_{M} .
$$

Now, $\Gamma\left(C^{N}, \omega_{C_{N}} \otimes\left(\Lambda^{k} \mathscr{I}_{S}\right)^{-1}\right)$ is a free $C\left[z_{1}, \ldots, z_{N}\right]$ module of rank 1 with the generator $\frac{d z_{1} \wedge \cdots \wedge d z_{N}}{f_{1}, f_{2} \cdots f_{k}}$, which is an eigenfunction with eigenvalue 
$\chi_{1}(g) \cdots \chi_{N}(g)\left(\delta_{1}(g) \cdots \delta_{k}(g)\right)^{-1}$. The $G$-structure of $\omega_{M}$ therefore corresponds to the trivial line bundle $M \times C$ with the $G$-action $G \times(M \times C) \rightarrow M \times C$ given by $(g,(y, \xi)) \rightarrow\left(g(y),\left(\chi_{1}^{-1} \cdots \chi_{N}^{-1} \delta_{1} \cdots \delta_{k}\right)(g) \xi\right)$. Our intent, of course, is to determine the canonical bundle on $X=M / G$. By Lemma $1, \omega_{X}=\left(p_{*} \omega_{M}\right)^{G}$, and hence we have

Lemma 2. $\omega_{X}=O_{X}\left[\chi_{1}^{-1} \cdots \chi_{N}^{-1} \delta_{1} \cdots \delta_{k}\right]$, where $O[\chi]$ for $\chi \in \operatorname{Hom}\left(G, C^{*}\right)$ is the coherent $O_{X}$-sheaf corresponding to the G-equivariant line bundle $M \times C$ over $M$ with the element $g \in G$ acting by $(y, \xi) \rightarrow(g(y), \chi(g) \xi)$ for $(y, \xi) \in M \times C$.

We can now immediately apply this result to varieties in weighted projective space. Consider a complete intersection in a single weighted projective space $W C P_{q_{1}, \ldots q_{N}}^{N-1}$. In the notation we have been using, this means that we take $G=C^{*}$, $V=C^{N}$ and the $G$ action $G \times V \rightarrow V$ given by $\left(\lambda,\left(z_{1}, \ldots, z_{N}\right)\right) \rightarrow\left(\lambda q_{1} z_{1}, \ldots, \lambda^{q_{N}} z_{N}\right)$. (We note that we may assume that the $q_{i}$ do not all have a nontrivial common factor.) We take $I_{S}$ to be the ideal in $C\left[z_{1}, \ldots, z_{N}\right]$ generated by a regular sequence of homogeneous elements with degrees $d_{1}, \ldots, d_{k}$, with $k$ being the codimension of $S$ in $C^{N}$. The algebraic variety $X=(S-[0]) / G$ is called a quasismooth weighted complete intersection of multidegree $\left(d_{1}, \ldots, d_{k}\right)$ in the weighted projective space $W C P_{q_{1}, \ldots, q_{N}}^{N-1}=C^{N} / G$. By our results above, the canonical sheaf of $X$ is given by $\omega_{X}=O_{X}\left(\Sigma d_{i}-\Sigma q_{j}\right)$.

The situation for a variety in a product of weighted projective spaces is conceptually equivalent, just a little notationally unwieldy. To describe a variety whose configuration matrix was described at the beginning of this section, we take $G=\left(C^{*}\right)^{l}$ and the characters $\chi_{m} \in \operatorname{Hom}\left(G, C^{*}\right) \equiv Z^{l}$, for $m=\left(m_{1}, \ldots, m_{l}\right)$ are of the form $\chi_{m}(\lambda)=\lambda_{1}^{m_{1}} \lambda_{2}^{m_{2}} \ldots \lambda_{l}^{m_{l}}$ for $\lambda=\left(\lambda_{1}, \ldots, \lambda_{l}\right) \in G$. In particular, since our embedding space is

$$
W C P_{q_{1}^{(1)}, \ldots, q_{N_{1}+1}^{(1)}}^{N_{1}} \times \ldots \times W C P_{q_{1}^{(1)}, \ldots, q_{N_{1}+1}^{(l)}}^{N_{l}}
$$

(with the $\left\{q_{i}^{(j)}\right\}_{i=1}^{N}{ }_{1}^{+1}$ not all having a common factor) we take $N=$ $\left(N_{1}+1\right)+\cdots+\left(N_{l}+1\right)$ and the linear action of $G$ on $C^{N}$ to have eigenvalues $m$ given by $\left(0,0, \ldots, 0, q_{i}^{(j)}, 0, \ldots, 0\right)$ for $1 \leqq i \leqq N_{j}+1$ and $1 \leqq j \leqq l$. With these definitions, $\prod_{n=1}^{l}\left(C^{N_{j}+1}-0\right) / G$ equals (3.16). Now consider a $G$-invariant cone $S$ in $C^{N}$ with ideal $I_{S}$ in $C\left[z_{1}, \ldots, z_{N}\right]$ generated by a regular sequence of $k$ eigenfunctions with eigenvalues $\left(d_{11}, d_{21}, \ldots, d_{l 1}\right), \ldots,\left(d_{1 k}, d_{2 k}, \ldots, d_{l k}\right)$. The algebraic variety $X=\left(S \cap \prod_{n=1}^{l}\left(C^{N_{j}+1}-0\right)\right) / G$ is called a quasismooth weighted complete intersection in (3.16). Reasoning as before, we have $\omega_{X}=O_{X}\left(\sum_{i=1}^{k} d_{1 i}\right.$ $\left.\sum_{j=1}^{N_{1}+1} q_{j}^{(1)}, \ldots, \sum_{i} d_{l i}-\sum_{j} q_{j}^{(l)}\right)$.

Having determined the structure of the canonical bundle of these varieties, we can finally invoke the results mentioned earlier to determine when we can resolve the singularities of these spaces to arrive at smooth varieties with vanishing first Chern class. In particular, if the dimension of $X$ is two or three and $\omega_{X}=O_{X}$, then there is a toroidal desingularization of $X$ having trivial canonical bundle [8]. For dimension two this is the minimal resolution; for dimension three this desingularization can be characterized as one of the minimal objects among all 
the toroidal desingularizations of $(X, x)$. These local minimal desingularizations of the singularities of $X$ can be patched together to obtain a desingularization $\tilde{X}$ of $X$ with $\omega_{\tilde{X}}$ being trivial, which will be called a minimal desingularization of $X$. Finally, by the criterion of the ampleness of invertible torus-invariant sheaves for a toroidal embedding [14] there is a line bundle over $\tilde{X}$ which is ample along the fibre direction of $\tilde{X} \rightarrow X$ near the exceptional divisors. Therefore $\tilde{X}$ is projective algebraic for projective algebraic $X$. Hence we have obtained the result:

Theorem. Let $G$ be an l-dimensional algebraic torus acting linearly on $C^{N}$ with eigenvalues $\chi_{s}, 1 \leqq s \leqq N$, where the $\chi_{s}$ 's are defined by $\chi_{1+} \sum_{x=1}^{\prime-1}\left(N_{x}+1\right)=\left(0,0, \ldots, q_{i}^{(j)}, 0, \ldots, 0\right)$. Let $S$ be a complete intersection in $C^{N}$ defined by $k$ eigenfunctions of $G$ with eigenvalues $\delta_{j}$ with $1 \leqq j \leqq k$. Assume that $S$ is nonsingular on $\left(S \cap \prod_{j=1}^{l}\left(C^{N_{j}+1}-0\right)\right)$. If $N-l-k$ is two or three and $\chi_{1} \cdots \chi_{N}=\delta_{1} \cdots \delta_{k}$, then the minimal desingularization $\tilde{X}$ of $X=\left(S \cap \prod_{n=1}^{l}\left(C^{N_{j}+1}-0\right)\right) / G$ has trivial canonical bundle. Furthermore, $\tilde{X}$ is projective algebraic whenever $X$ is projective algebraic.

For a quasismooth weighted complete intersection $X$ in a product of weighted projective spaces (of dimension 2 or 3 ), so long as

$$
\sum_{j=1}^{k} d_{i j}=\sum_{j=1}^{N_{i}+1} q_{j}^{(j)}
$$

then the minimal desingularization $\tilde{X}$ of $X$ is a smooth projective Calabi-Yau manifold.

\section{Resolved Cohomology}

In the previous section, we have determined the conditions on a complete intersection in a product of weighted projective spaces to admit a desingularization to a manifold of vanishing first Chern class. Of course, as far as string theory goes, it is well known that we do not have to resolve such singular spaces for string propagation to make sense; on the other hand, the string nonetheless probes the resolved topology. In particular, the spectra of the massless states is determined by the cohomology of the resolved manifold. For example, the Euler number (for threefolds), as originally shown in [15], determines the number of massless generations of particles. In this section we study the desingularizations invoked in the last section in a bit more detail in order to extract a simple formula for the Euler number of the resolved space. We also compare our result with that found by Vafa using semiclassical arguments applied to Landau-Ginzburg models. This serves to illustrate the correspondence between twisted sectors and blowing-up modes.

For concreteness and simplicity we shall restrict attention for the moment to three-folds which are hypersurfaces in a weighted projective four space. Even for this relatively restricted set of possibilities, there are thousands of examples which meet the Calabi-Yau condition, as we shall make more clear in the next section. (One should compare this with the fact that when one restricts to 
Calabi-Yau hypersurfaces in ordinary projective space $C P^{4}$, there is only one example.) We shall also restrict our attention, for now, to examples which are completely quasismooth. This means that $X \cap\left\{z_{i}=0 \mid i \in I\right\}$ are quasismooth for $I \subseteq\left\{j \mid q_{j}>1\right\}$ (i.e. meet the transversality constraint). It is not hard to show that if we denote by $\pi$ the map $\pi: C P^{4} \rightarrow W C P_{q_{1}}^{4}, \ldots, q_{5}$ then $X$ in $W C P^{4}$ is completely quasismooth if and only if $Y=\pi^{-1}(X)$ is nonsingular in $C P^{4}$. For example, all Fermat hypersurfaces meet this constraint. The formula we shall derive is the following:

Let $X$ be a completely quasismooth hypersurface in $W C P_{q_{1}, \ldots, q_{5}}^{4}$ with degree $d=\Sigma q_{i}$ and assume $\left(q_{1}, \ldots, q_{i-1}, q_{i+1}, \ldots, q_{5}\right)=1$ for all $i$. Let $Y=\pi^{-1}(X)$, $Y_{j}=Y \cap\left\{\zeta_{j}=0\right\}$, where $\zeta_{j}$ is a homogeneous $C P^{4}$ coordinate and let $Y_{I}=Y$ if $I=\phi$ and $\cap_{i \in I} Y_{i}$ for $I \subset\{1,2,3,4,5\}$. Assuming that $Y_{j}$ are codimension one submanifolds of $Y$ and intersect transversely, we have

$$
\chi(\tilde{X})=\sum_{I, Y_{I} \neq \varnothing}\left((-1)^{|I|} \sum_{J \subset I}(-1)^{|J|} \frac{c_{J}}{m_{J}}\right) \chi\left(Y_{I}\right) .
$$

In this expression, $c_{I}=$ g.c.d. $\left(q_{j} \mid j \notin I\right)$ and $m_{I}=\frac{\prod_{j \notin I} q_{j}}{c_{I}}$ if $Y_{I} \neq \varnothing$ and zero otherwise.

Notice that this formula gives the Euler number of the resolved space in terms of Euler numbers of subvarieties of ordinary projective space. The latter, of course, are readily computed using standard methods. To prove this, we need one simple lemma.

Lemma. Let $M$ be a compact complex manifold and $M_{1}, \ldots, M_{t}$ be pure codimension 1 submanifolds of $M$ such that any finite number of them meet transversely. Let $\pi: M \rightarrow W$ be a proper holomorphic map (i.e. the inverse image of a compact set is compact, thus ensuring that the fiber of this map is compact) from $M$ to a normal analytic space $W$, with finite fibers. For a subset I of $\{1,2, \ldots, t\}$ denote $M_{I}=\bigcap_{i \in I} M_{i}$, and $W_{I}=\pi\left(M_{I}\right)$. Assume that for each $I, \pi^{-1}\left(W_{I}\right)=M_{I}, W_{I}-\bigcup_{I \subsetneq J} W_{j}$ are smooth and that $\pi_{\text {rest }}: M_{I}-\bigcup_{I \mp J} M_{I} \rightarrow W_{I}-\bigcup_{I \mp J} W_{J}$ is an $m_{I}$-fold unramified cover. Then

$$
\chi\left(W_{K}\right)=\sum_{K \subset I} b_{K I} \chi\left(M_{I}\right)
$$

where

$$
b_{K I}=(-1)^{|I|} \sum_{K \subset J \subset I ; m_{J} \neq 0}(-1)^{|J|} m_{J}^{-1} .
$$

The proof of this lemma is a straightforward exercise in induction on $|K|$ (from $|K|=t$ to $|K|=0$ ); we will not write out the details.

We now use this to derive (4.1). Denote the birational morphism from $\tilde{X}$ to $X$ by $\sigma: \tilde{X} \rightarrow X$. Set $\chi(\varnothing)=0$. We regard $X$ as the quotient of $Y$ by the diagonal action $\left(\mu_{q_{1}}, \ldots, \mu_{q_{5}}\right)$. Since the $Y_{I}$ are all invariant under this action, the natural projection $\pi: Y \rightarrow X$ and the $Y_{j}$ 's satisfy the assumption of the lemma above with 
$m_{I}=\frac{\prod_{j \notin I} q_{j}}{c_{I}}$. Now, if $p$ is an element of $Y_{I}-\bigcup_{j \ngtr I} Y_{J}$, it is easy to see that isotropy subgroup at $p$ is isomorphic to $\prod_{i \in I} \mu_{q_{1}} \times \mu_{c_{I}}$. Therefore $\pi_{\text {rest }}: Y_{I}-\bigcup_{J \ngtr I} Y_{j} \rightarrow X_{I}-$ $\bigcup_{J \supsetneq I} X_{J}$ is a principle bundle with group having order $\prod_{j \notin I} q_{j} / c_{I}$. Furthermore,

$$
(X, \pi(p)) \cong\left(\mathbf{C}^{|I|} / \mu_{c_{I}} \times \mathbf{C}^{3-|I|},[0] \times 0\right) .
$$

In this expression, $\eta \in \mu_{c_{I}}$ acts on $\mathbf{C}^{|I|}$ diagonally with eigenvalues $\eta^{q_{\mathrm{t}}}, \quad i \in I$. Since $\left(q_{1}, \ldots, q_{i-1}, q_{i+1}, \ldots, q_{5}\right)=1$ for all $1 \leqq i \leqq 5$, and $\omega_{X}$ is trivial, then $X$ is singular at $\pi(p)$ and $\prod_{j \in I} \eta^{q_{i}}=1$ when $c_{I}>1$. By the construction of toroidal desingularization $\tilde{X}$, we can conclude that: $\chi\left(\sigma^{-1}(x)\right)=c_{I}$ for $x \in X^{I}-\bigcup_{J \ngtr I} X_{J}[8]$. Hence,

$$
\chi\left(\bigcup \sigma^{-1}\left(X_{j}\right)\right)=\sum_{I \neq \varnothing} \chi\left(\sigma^{-1}\left(X_{I}-\bigcup_{J \supsetneq I} X_{J}\right)\right)
$$

which equals

$$
=\sum_{I \neq \varnothing} c_{I} \chi\left(X_{I}-\bigcup_{J \ngtr I} X_{J}\right)
$$

After a bit of algebra, this yields

$$
\chi\left(\bigcup \sigma^{-1}\left(X_{j}\right)\right)=\sum_{K \neq \varnothing}\left(\sum_{\varnothing \neq I \subseteq K}(-1)^{|K|-|I|} c_{I}\right) \chi\left(X_{K}\right) .
$$

From this we therefore have

$$
\chi(\tilde{X})=\chi(X)+\sum_{2 \leqq|K|}\left((-1)^{|K|}+\sum_{\emptyset \neq I \subseteq K}(-1)^{|K|-|I|} c_{I}\right) \chi\left(X_{K}\right) .
$$

Now, by our previous lemma and (4.8), we therefore have

$$
\chi(\tilde{X})=\sum_{I} b_{\circledast I} \chi\left(Y_{I}\right)+\sum_{2 \leqq|J|}\left(\sum_{2 \leqq|K|, K \subseteq J}\left((-1)^{|K|}+\sum_{ø \neq I \subseteq K}(-1)^{|K|-|I|} c_{I}\right) b_{K J}\right) \chi\left(Y_{J}\right) .
$$

From this expression our theorem follows directly.

To illustrate our formula, consider the corollary which follows by restricting attention to the special case of a hypersurface $X$ in $W C P_{q_{1}}^{4}, \ldots, q_{s}$, with the degree $d$ of $X$ satisfying the condition $d=\Sigma q_{i}$. Let $Y$ be the hypersurface $\pi^{-1}(X)$ in $C P^{4}$, where $\pi: C P^{4} \rightarrow W C P_{q_{1}, \ldots, q_{5}}^{4}$ is the map

$$
\left(y_{1}, \ldots, y_{5}\right) \in C P^{4} \rightarrow\left(y_{1}^{q_{1}}, \ldots, y_{5}^{q_{5}}\right) .
$$

If $Y$ is nonsingular and the set of all $y_{j}=0$ intersect $Y$ transversely, then our 
theorem reduces to the following simple formula for the Euler characteristic of $\tilde{X}$ :

$$
\chi(\tilde{X})=\frac{d}{q_{1}, \ldots, q_{5}}\left(-d \sum_{i<j} q_{i} q_{j} c_{i j}^{2}+\sum_{i<j<k} q_{i} q_{j} q_{k} c_{i j k}^{2}\right) .
$$

Again, $c_{I}=$ g.c.d. $\left(q_{j} \mid q_{j} \notin I\right)$. Notice that as a consequence, the Euler number depends only on the $q_{i}$.

The reader can check that this simple formula reproduces all of the results of $[2,6,5,7,9]$.

\section{Conclusions}

In this letter we have put the analyses surrounding Calabi-Yau vacua constructed in weighted projective space on a more firm mathematical footing. The general argument of [2] in conjunction with the extensive numerical verification in $[2,5,6,7]$ left little room to doubt the veracity of the results and it is satisfying to see that our mathematical analysis provides further confirmation. Furthermore, our Euler number formula provides an efficient means of analysing specific examples.

It is important to note that the analyses of [2] and [3] associate a CalabiYau manifold with an orbifold of a Landau-Ginzburg theory as reviewed in the first section of this letter. An interesting and unsolved question is whether a geometrical interpretation can be ascribed to the Landau-Ginzburg theory itself. We have no firm result in this area, but would like to state some evidence which leads to a natural candidate. More precisely, we now give a geometric interpretation to the whole chiral ring of primary fields in a Landau-Ginsburg theory; this goes beyond the known interpretation for the integrally charged members of this ring. form

In [16], the author investigates the geometrical significance of rings of the

$$
C\left[z_{0}, \ldots, z_{n}\right] /\left(\partial W / \partial z_{i}\right)
$$

where $W$ is a quasihomogeneous function on $\mathbf{C}^{\mathbf{n}+\mathbf{1}}$.

Notice that this is nothing but the ring of chiral primary fields of a LandauGinzburg theory [17]. In particular, let the conformal weights of the fields $z_{i}$ be called $h_{i}=w_{i} / 2$ and their $U(1)$ charges be $w_{i}$. To understand the result of [16] we need a few definitions. Let $V \subset \mathbf{C}^{\mathbf{n}+1}$ be the affine variety defined by the euation $W=1$ and let $w_{i}=u_{i} / v_{i}$ with $\left(u_{i}, v_{i}\right)=1$ and $d=1$.c.m. $\left(v_{0}, \ldots, v_{n}\right)$. We denote $q_{i}=d w_{i}$, with $q_{i} \in Z$. Let the monomial in the ring (5.1) $z_{0}^{\alpha_{0}} z_{1}^{\alpha_{1}} \ldots z_{n}^{\alpha_{n}}$ (with the $\alpha_{i}$ all natural numbers) be denoted by $z^{\alpha}$, where $\alpha=\left(\alpha_{0}, \ldots, \alpha_{n}\right)$. Finally, we define a function $l(\alpha)$ by $l(\alpha)=\sum_{i=0}^{n}\left(\alpha_{i}+1\right) w_{i}$. Notice that up to integer shifts, $l(\alpha)$ computes the $U(1)$ charge of $z^{\alpha}$.

With these definitions, we then have the result that

$$
H^{n}(V, C) \cong\left[z_{0}, \ldots, z_{n}\right] /\left(\partial W / \partial z_{i}\right)
$$


and

$$
H^{n-1}(X, C)_{0} \cong \text { Subspace of (5.2) with integer } l(\alpha) .
$$

In this latter equation, $X=\left\{[z] \in W C P_{q_{1}, \ldots, q_{n}}^{n} \mid W([z])=0\right\}$, i.e., $X$ is a variety in weighted projective space. (We note that the subscript in $H^{n-1}(X, C)_{0}$ refers to the primitive part of the cohomology; in the case $n=4$ for example, this subscript can be dropped.)

In other words, (5.2) gives a cohomological interpretation to the whole chiral primary ring of the (unorbifolded) Landau-Ginsburg theory in terms of a noncompact affine variety. Furthermore, (5.3) gives the familiar cohomological interpretation to the integrally charged members of the chiral ring in terms of a compact algebraic variety in weighted projective space.

For example, consider the simplest example of the quintic $Y_{4 ; 5}$ in $C P^{4}$. The chiral ring (5.1) then consists of elements of the form

$$
\left(1 ; P_{1}([z]), P_{2}([z]), \ldots, P_{15}([z])\right),
$$

where $P_{n}([z])$ is an $n^{\text {th }}$ order monomial in the $z_{i}$ in which no individual $z_{i}$ occurs with a power exceeding three (since in (5.1) we quotient by the fourth powers of the monomials). Some time $\mathrm{ago}^{3}[18]$ it was elegantly shown that the subset of elements consisting of $\left(1, P_{5}([z]), P_{10}([z]), P_{15}([z])\right)$ are representatives for the third cohomology group $H^{3}\left(Y_{4.5}, C\right)$. Notice that these elements are precisely those which have integral $U(1)$ charges and hence integral $l(\alpha)$. Thus, we see the interpretation found in [18] also emerging from (5.3). These elements of (5.1) are, of course, precisely those which survive the orbifolding operation discussed in Sect. 1 which takes us from the original Landau-Ginsburg theory to string propagation on a Calabi-Yau manifold.

Now we can go further. All of the elements of (5.4), not just the zeroth, fifth, tenth and fifteenth order terms, can be interpreted as the fourth cohomology $H^{4}(V, C)$, where $V$ is the affine variety

$$
z_{1}^{5}+z_{2}^{5}+z_{3}^{5}+z_{4}^{5}+z_{5}^{5}=1 \subset C^{5} .
$$

This description is unified by the observation that our compact algebraic variety may be viewed as the boundary of the noncompact affine variety $V$ at infinity.

This reasoning leads to the speculation that the original Landau-Ginsburg theory has a natural geometric interpretation in terms of $V$; work along these lines is in progress.

Acknowledgements. We thank C. Vafa for many useful discussions.

\section{References}

1. Gepner, D.: Phys. Lett. B199, 380 (1987)

2. Greene, B. R., Vafa, C., Warner, N. P.: Nucl. Phys. B324, 371 (1989)

\footnotetext{
3 This is simply an example of the general cohomological interpretations found in [18]
} 
3. Martinec, E.: In: V. G. Knizhnik Memorial Volume (Brink, L. et al. (eds.)). Phys. Lett. 217B, 431

4. Dixon, L., Harvey, J., Vafa, C., Witten, E.: Nucl. Phys. B261, 620 (1985); B274, 285 (1986)

5. Kim, J., Park, C., Yoon, Y.: Calabi-Yau spaces and rescale invariant $N=2$ minimal superconformal theories, KAI preprint, 1989

6. Lynker, M., Schimmrigk, R.: A-D-E Calabi-Yau Manifolds, Texax Preprint 1989

7. Fuchs, J., Klemm, A., Scheich, C., Schmidt, M.: Heidelberg preprint, HD- THEP-89-16

8. Roan, S.-S., Yau, S.-T.: Acta Mathematica SINICA, New Series, 1987, Vol 3. (3) 256

9. Greene, B. R.: Superconformal compactifications in weighted projective space Commun. Math. Phys. 130, 335-355 (1990)

10. Vafa, C.: Mod. Phys. Lett. A4 1169 (1989); Mod. Phys. Lett. A4, (1989) 1615

11. Zamolodchikov, A. B.: Sov. J. Nucl. Phys. 44, 529 (1986)

12. Vafa, C., Warner, N. P.: Phys. Lett. 218B, 51 (1989)

13. Hubsch, T.: Commun. Math. Phys. 108, 291 (1987); Green, P., Hubsch, T.: Commun. Math. Phys. 109, 99 (1987)

14. Kempt, G., Knudsen, F., Mumford, D., Saint-Donat, B.: Toroidal Embedding 1. Lecture Notes, Vol. 339. Berlin, Heidelberg; New York: Springer

15. Candelas, P., Horowitz, G., Strominger, A., Witten, E.: Nucl. Phys. B258, 46 (1985)

16. Steenbrink, J.: Composito Mathematica, Vol. 34, Fasc 2, 211 (1977)

17. Lerche, W., Vafa, C., Warner, N. P.: Nucl. Phys. B324, 427 (1989)

18. Candelas, P.: Nucl. Phys. B298, 458 (1988)

Communicated by A. Jaffe 
